\title{
Multiply-Charged Negative Clusters of Adenosine-5'-Monophosphate in the Gas Phase
}

\author{
Bo Liu, Preben Hvelplund and Steen Brøndsted Nielsen \\ Department of Physics and Astronomy, University of Aarhus, Aarhus, Denmark
}

\begin{abstract}
Multiply-charged noncovalent cluster anions of adenosine-5'-monophosphate (AMP) were formed by electrospray ionization (ESI). Ions in higher charge states were observed when the ions were accumulated in an ion trap with helium buffer gas before detection. We determined the smallest size $\left(n_{a}\right)$ or appearance size as a function of charge state $(q)$, i.e., $n_{a}=4$ for $q=2$, $n_{a}=8$ for $q=3$, and $n_{a}=13$ for $q=4$. The relation between $n_{a}$ and $q$ can be described by a charged droplet model. When the size is larger than $n_{a}$ for a given $q$, the fragmentation pathway of an anion cluster is dominated by loss of neutral fragments. In contrast, when the size approaches the appearance size, only charged fragments are formed. (J Am Soc Mass Spectrom 2005, 16, 1840-1845) (c 2005 American Society for Mass Spectrometry
\end{abstract}

C lusters are interesting systems since they bridge the gap between gas-phase and liquid phase $[1,2]$. Cluster cations such as metal clusters have been extensively studied during the last two decades [3, 4]. Recently, the study of biomolecular cluster cations has become an active research field, and much work has dealt with the formation, dissociation, and charge partitioning among fragments of protein cluster cations [5-10]. Magic numbers for amino acid cationic clusters [11-13] and preference for homochirality [14, 15] have been reported. Negatively charged clusters of amino acids have also been produced [13].

The formation and energetics of gas-phase multiplycharged anions (MCA) is a subject that has attracted much attention [16-18]. Even though several multiplycharged molecular anions have been measured, observations of multiply-charged cluster anions are few. Multiply-charged metal cluster anions were observed by electron attachment to monoanions in a Penning trap [19-22] and by laser ablation of metal surfaces [23]. Small dianions of carbon clusters were formed by $\mathrm{Cs}^{+}$ ion sputtering of graphite [24-26]. Electrospray ionization (ESI) produced multiply-charged cluster anions of supramolecules, $\left[\mathrm{X}^{+}\right]_{n}\left[\mathrm{~A}^{-}\right]_{m}[27]$ where $\mathrm{X}$ for example is an alkali and $\mathrm{A}$ is a halide. Fullerene dianions were observed by laser desorption [28], electron attachment to monoanions $[29,30]$ and electrospray of a solution that contained fullerene and an organic electron donor [31]. Here we report, to our knowledge, the first observation of negatively, multiply-charged noncovalent clusters made of adenosine $5^{\prime}$-monophosphate (AMP). The dependence of cluster size on charge state was

Published online September 26, 2005

Address reprint requests to Dr. B. Liu, Department of Physics and Astronomy, University of Aarhus, Aarhus, Denmark. E-mail: liubo@phys.au.dk measured, and the results, are modeled by a charged droplet model. The fragmentation pathways of multiply-charged negative clusters are also presented.

\section{Experimental}

Two setups were applied in the experiments: an electrostatic ion storage ring (ELISA) and an accelerator mass spectrometer (AMS). In both setups, negative clusters of AMP were produced by ESI. A saturated solution of AMP in methanol was sprayed.

Detailed information about the ESI source (Figure 1a) is described in reference [32]. Briefly, the source is mounted on the high-voltage platform of the accelerator. A stainless steel hypodermic needle is, via a fused silica capillary, connected to a syringe containing a solution of the analyte. A syringe pump (Harvard apparatus) delivers a constant flow through the needle. A typical flow rate is $1 \sim 2 \mu \mathrm{l} / \mathrm{min}$. A voltage of 3 to $4 \mathrm{kV}$ relative to the heated capillary is put on the needle. The capillary is normally heated for desolvation of solvent molecules from analyte molecules. At the exit of the capillary, ions emerge into the first vacuum zone where a pressure of around $1 \mathrm{mbar}$ is maintained. The ions focused by a tube lens go through a skimmer into a second region. The voltage on the tube lens varies from 50 to $200 \mathrm{~V}$. The pressure in the second region is further reduced to $10^{-3}$ mbar. The ions are steered through this section by an octopole beam guide, and enter the third vacuum region through lenses. This region is evacuated to $10^{-5}$ mbar. An Einzel lens assembly focuses the ions into the acceleration region.

The ESI source used for ELISA is equipped with a 22-pole ion trap that was constructed according to the design of Gerlich $[33,34]$. The trap is mounted in the third vacuum region. Ions can be accumulated in the 


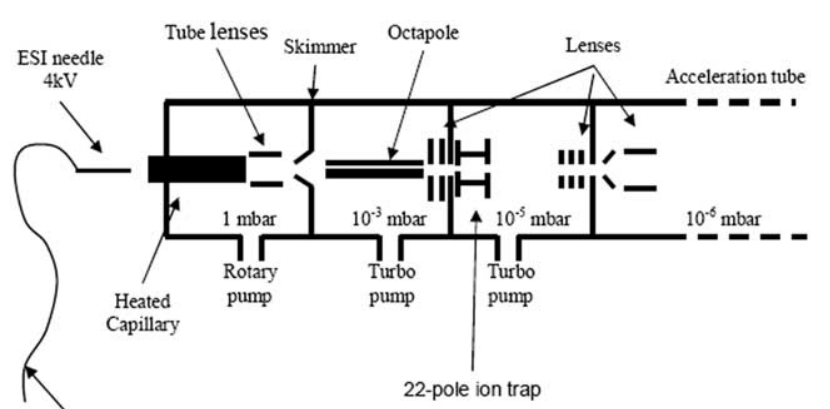

(a)

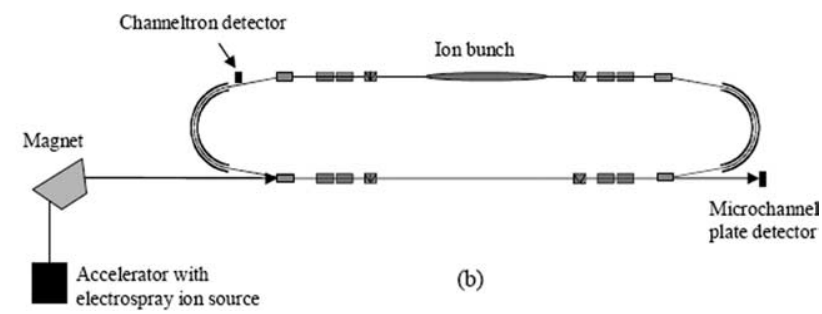

Figure 1. Schematic drawing of (a) the ESI source and (b) the electrostatic ion storage ring ELISA.

ion trap or allowed to pass through directly. When trapping and accumulating ions, the trap was filled with helium buffer gas (typically $10^{-2}$ mbar) while in the continuous beam mode the trap acted as an optical lens to guide and focus the ions.

After exiting the source, ions were accelerated to an energy of $22 \times q \mathrm{keV}$, selected by a bending magnet and injected into ELISA. The ring consists of two straight sections about $3 \mathrm{~m}$ long; the ions were deflected at the ends by two parallel $10^{\circ}$ deflectors and a $160^{\circ}$ cylindrical deflector. A multichannel plate (MCP) detector is placed at the end of one straight section. A channeltron detector is placed just after one of the $10^{\circ}$ deflectors and is $1 \mathrm{~cm}$ away from the track of the circulating beam (Figure 1) $[35,36]$. When there were no voltages on the $10^{\circ}$ deflectors, the stored ion beam hit the MCP and thus by scanning the magnet a mass spectrum was taken. For lifetime measurements, we accumulated ions in the trap for about $100 \mathrm{~ms}$ and ions in a bunch were accelerated and injected into ELISA, where they were stored. The MCP was in this case used to detect neutral fragments that were formed in the straight section on the injection side of the ring. Charged fragments with $m / q$ ( $m$ being the mass of the ion) higher than the parent ions were measured by the channeltron.

The experimental setup for the accelerator mass spectrometer has been described in detail elsewhere [37]. Briefly, after acceleration, an $\mathrm{m} / \mathrm{q}$ analysis was performed by a large, $2-\mathrm{m}$ radius, $72^{\circ}$ bending magnet capable of deflecting singly charged ions with mass up to $5000 \mathrm{u}$. After magnetic analysis the mass-selected ions passed through a target gas contained in a $3-\mathrm{cm}$ long differentially pumped cell with entrance and exit apertures of 1 and 3-mm in diameter, respectively. Then the ions entered a computer-controlled $180^{\circ}$ hemispher- ical electrostatic analyzer (ESA) with a radius of $15 \mathrm{~cm}$. The mass resolution is typically about 500 .

\section{Results and Discussion}

\section{Observations of Multiply-Charged Anion Clusters}

Mass spectra of ions produced in the ion source were recorded at ELISA, and the currents of AMP clusters were measured under three different ion source conditions: (1) heated capillary temperature of $170{ }^{\circ} \mathrm{C}$ and trap in pulsed mode, (2) heated capillary temperature of $60^{\circ} \mathrm{C}$ and trap in continuous mode, and (3) heated capillary temperature of $60^{\circ} \mathrm{C}$ and trap in pulsed mode. The signal from AMP clusters measured under conditions 1, 2, and 3 are shown in Figure $2 a, b$, and $c$, respectively. Figure 2a reveals that only singly charged clusters were measured under condition 1 . From a comparison of Figure $2 b$ and Figure 2c, it is seen that peaks can be assigned to multiply-charged clusters when the ions are trapped and that intensities of multiply-charged cluster anions are higher than the intensities when the ions are not trapped. The measured signal of the AMP monoanion was about $10^{6}$ counts per $\mathrm{s}$ and those of multiply-charged clusters like (AMP) ${ }_{10}^{3-}$ and $(\mathrm{AMP})_{15}^{4-}$ were $10^{4}$ and $10^{3}$ counts per $\mathrm{s}$, respectively.

The ions are formed in the ion source and not in the trap, since the Coulomb repulsion between ions pre-

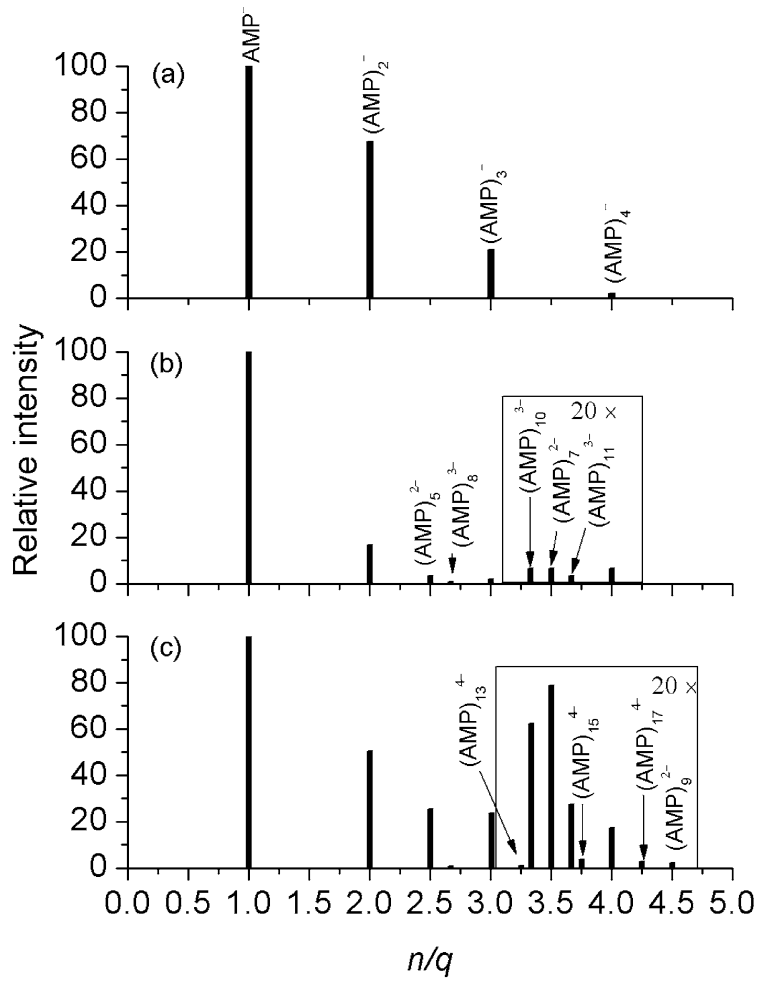

Figure 2. Relative intensities of noncovalent AMP cluster anions. (a) Capillary temperature $170{ }^{\circ} \mathrm{C}$ and pulsed beam. (b) Capillary temperature $60^{\circ} \mathrm{C}$ and continuous beam. (c) Capillary temperature $60^{\circ} \mathrm{C}$ and pulsed beam. 


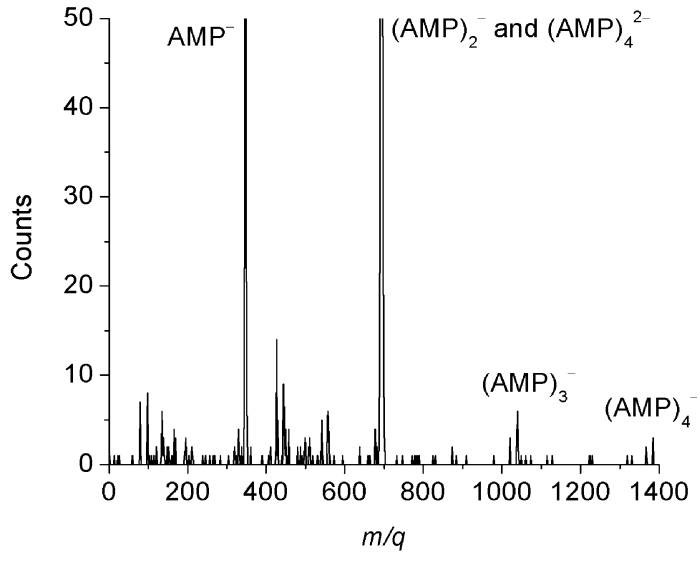

Figure 3. CID spectrum obtained after collisions between a mixed beam of $(\mathrm{AMP})_{2}^{-}$and $(\mathrm{AMP})_{4}^{2-}$ and neon.

vents ion-ion reactions, and furthermore, the ion concentration is low. The importance of the trap is to cool down the ions in collisions with the helium buffer gas. In the continuous ion mode, there is no way to dissipate or lose the excess energy and therefore "hot" ions dissociated before detection.

The smallest number of constituents of an observed cluster depends on its charge state and is five for a dianion, eight for a trianion, and 13 for a tetranion (Figure 2c). However, from Figure 2 only, we cannot tell whether the peak marked as a dimer monoanion has some contribution from a tetramer dianion because they both have the same mass-to-charge ratio $(m / q=693)$. A collision induced dissociation (CID) experiment was therefore carried out with an accelerator mass spectrometer in which ions with $m / q=693$ were selected and collided with Ne. A fragmentation spectrum is shown in Figure 3, and it is obvious that there are fragments of $\mathrm{m} / \mathrm{q}$ larger than 693 that correspond to the singly charged trimer and tetramer, which shows that the tetramer dianion indeed exists. Also, the tetramer monoanion is formed by electron loss from the dianion in the collision process.

Several isomers of the clusters exist and to obtain some information on the possible structures, we carried out B3LYP/6-31 + G(d)//PM3 quantum chemical calculations with the Gaussian 98 [38] program package. The strongest hydrogen bond involves the negatively charged oxygen of a phosphate group. In the case of dimers, three possible isomer structures were calculated where there is a hydrogen bond between $\mathrm{O}^{-}$and $\mathrm{OH}$ of the phosphoric acid group $\left(\mathrm{OH}_{\text {phos }}\right)$ (Figure $\left.4 \mathrm{~b}\right), \mathrm{b} \mathrm{O}^{-}$ and $\mathrm{OH}$ of the sugar $\left(\mathrm{OH}_{\text {sugar }}\right)$, and $\mathrm{O}^{-}$and $\mathrm{NH}$ of adenine $\left(\mathrm{NH}_{\text {base }}\right)$. The calculations show that the former is most stable by at least $0.5 \mathrm{eV}$. Based on the dimer structure (Figure $4 \mathrm{~b}$ ), three trimer structures were generated by hydrogen bonds between $\mathrm{O}^{-}$and $\mathrm{OH}_{\text {phos }}$ (Figure $4 \mathrm{c}$ ), $\mathrm{O}^{-}$and $\mathrm{OH}_{\text {sugar }} \mathrm{O}^{-}$and $\mathrm{NH}_{\text {base }}$ of the third AMP molecule. The former is the most stable one by 0.3 $\mathrm{eV}$. Hence the calculations indicate that the most stable isomers are formed by hydrogen bonds between phosphate and phosphoric acid groups. The dimers and trimers are then difficult to further deprotonate to form

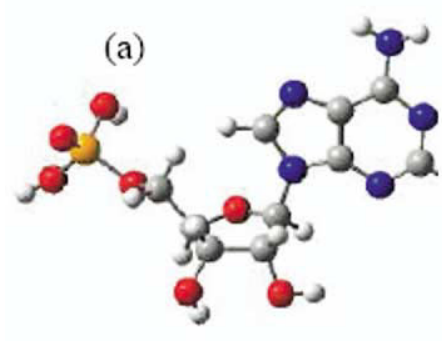

(b)
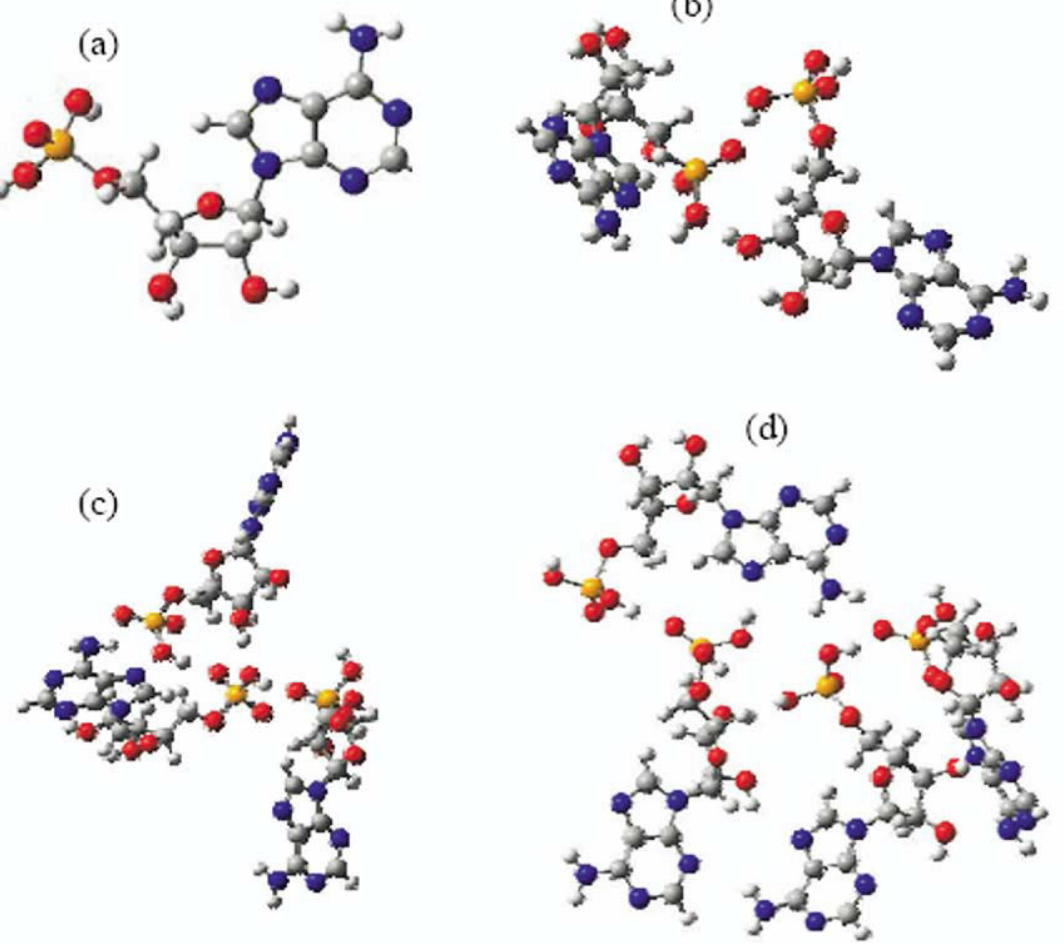

Figure 4. Possible structures of the singly charged (a) monomer, (b) dimer, (c) trimer, and (d) tetramer. 


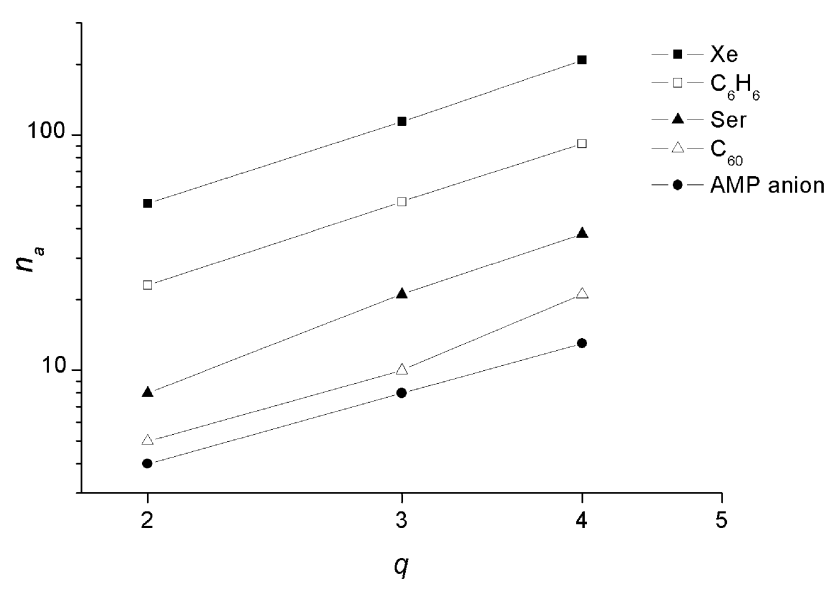

Figure 5. Stability diagram. The $\log -\log$ plot shows that $n_{a}$ scales approximately with $q^{2}$ for positive and negative (AMP) cluster ions.

a dianion, since the distance between the phosphate groups becomes too small. Several tetramers are generated based on the trimer shown in Figure 4c. However, the situation is different since no more hydrogen bonds can be formed between $\mathrm{O}^{-}$and another $\mathrm{OH}$ or $\mathrm{NH}$, and the calculated most stable tetramer (Figure $4 \mathrm{~d}$ ) has a structure different from that of the dimer and trimer where the phosphate groups are close to each other. In this case, the distance between the phosphoric groups is larger $(\sim 10 \AA)$, and therefore, a dianion is more likely to be formed.

The smallest size of a cluster with charge $q$ is called the appearance size of the cluster $n_{a}$. To understand the relationship between $n_{a}$ and $q$ information about the structures of the multiply-charged clusters is useful. For the large clusters we apply a charged droplet model to relate $n_{a}$ to $q$, which has earlier been used to describe cluster cations [39-41]. In this model, a cluster is approximated by a charged spherical droplet and detailed information about structure is neglected. The appearance size is determined from a balance between the Coulomb repulsion and the surface tension, which leads to the Rayleigh stability limit defined by:

$$
R_{a}^{3}=q^{2} e^{2} /(16 \pi \gamma)
$$

where $\gamma$ is the surface tension and $R_{a}$ is the radius of the droplet. Since $R_{a}^{3}$ is proportional to $n_{a}$, this leads to $n_{a} \propto q^{2}$.

In Figure 5, the appearance size $n_{a}$ of AMP clusters is plotted as a function of charge state $q$ together with some atomic and molecular clusters. It should be noted that it is a log-log plot, so the relation appears to be a straight line. $n_{a}$ of AMP clusters is smaller than that of other clusters of the same charge state, which is likely attributable to the formation of several hydrogen bonds between AMP constituents and the large size of an AMP molecule.

\section{Dissociation of Multiply-Charged Anion Clusters}

Dissociation lifetimes of multiply-charged clusters were studied in the storage ring ELISA. The decay spectrum of $(\mathrm{AMP})_{7}^{2-}$ is shown in Figure 6a. The neutral fragments measured by the MCP detector in the first $20 \mathrm{~ms}$ originated from decay of metastable ions that were created by collisions with the residual gas in the source when the ions were exiting the ion trap. In ELISA, neutral fragments were formed by collisions with the residual gas but this happened on a second timescale and therefore only contributed to the "constant background" in the decay spectrum. The decay spectra shown in Figure 6a are not exponential but follow a power-law decay, $t^{-1}$. A power-law decay has also been observed for small clusters, fullerenes, and amino acids [42-44]. This occurs when the internal energy distribution of the metastable clusters is broad due to collisional

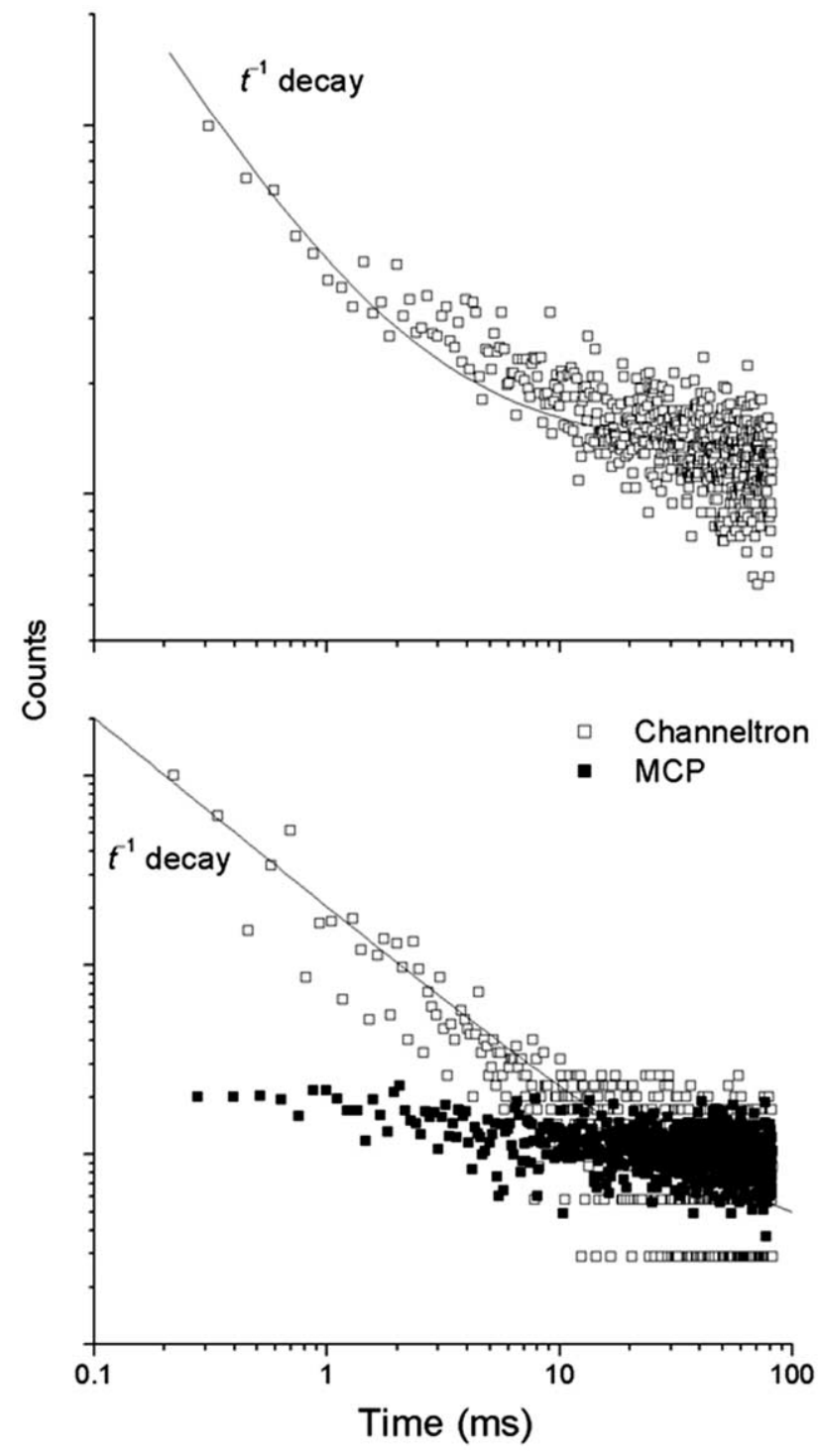

Figure 6. Decay spectra of the dianion of $(\mathbf{a})(\mathrm{AMP})_{7}$ detected by the MCP and (b) (AMP) $)_{5}$ detected by the MCP (filled squares) and channeltron (open squares). 
activation. The power-law decay, therefore, is independent on the emitted particles, whether they are neutral or charged (see detailed information in [4]).

Decay spectra of $(\mathrm{AMP})_{5}^{2-}$ are shown in Figure $6 \mathrm{~b}$. The filled squares indicate the neutral products detected by the MCP while the open squares indicate the charged products detected by the channeltron. The decay curve for charged fragments follows a $t^{-1}$ decay law, again due to a broad distribution of internal energy, while the decay curve for neutral fragments is flat because neutral fragments from metastable ions are few and most neutral fragments are formed from collisions with the residual gas in the ring. It is seen that charged fragments dominate over neutral fragments in the case of dissociation of $(\mathrm{AMP})_{5}^{2-}$.

From a comparison of the decay channels of $(\mathrm{AMP})_{7}^{2-}$ and $(\mathrm{AMP})_{5}{ }^{2-}$, we propose that for a given $q$, if the size is much larger than the appearance size, neutrals evaporate from the cluster until the cluster approaches the Rayleigh limit and then "fission" dominates to give charged fragments. Similar fragmentation pathways were observed in the case of metal, amino acid and peptide cation clusters $[4,45]$.

\section{Conclusions}

We have formed multiply-charged noncovalent cluster anions by electrospray ionization. By using an ion trap and low capillary temperature, more multiply-charged clusters were observed. The appearance size $n_{a}$ for a given charge state $q$ was found to be proportional to $q$ [2], in agreement with the charged droplet model. The multiply-charged clusters dissociate via evaporation of the neutral constituents when the size is larger than $n_{a}$. Fission leading to fragments of charged constituents occurs when the cluster size approaches $n_{a}$.

\section{Acknowledgments}

This work was supported by the Danish National Research Foundation through the Aarhus Center for Atomic Physics (ACAP). SBN acknowledges support from FNU. The authors thank Professors J. U. Andersen and J. S. Foster for many valuable discussions.

\section{References}

1. Castleman, A. W.; Keesee, R. G. Clusters-Bridging the Gas and Condensed Phase. Acc. Chem. Res. 1986, 19, 413-419.

2. Castleman, A. W.; Bowen, K. H. Clusters: Structure, Energetics, and Dynamics of Intermediate States of Matter. J. Phys. Chem. 1996, 100, 12911-12944.

3. de Heer, W. A. The Physics and Simple Metal Clusters: Experimental Aspects and Simple Models. Rev. Mod. Phys. 1993, 65, 611-676.

4. Näher, U.; Bjørnholm, S.; Frauendorf, S.; Garcias, F.; Guet, C. Fission of Metal Clusters. Phys. Rep. 1997, 285, 245-320.

5. Schwartz, B. L; Bruce, J. E.; Anderson, G. A.; Hofstadler, S. A.; Rockwood, A. L.; Smith, R. D.; Chilkoti, A.; Stayton, P. S. Dissociation of Tetrameric Ions of Noncovalent Streptavodin
Complexes Formed by Electrospray-Ionization. J. Am. Soc. Mass Spectrom. 1995, 6, 459-465.

6. Fitzgerald, M. C.; Chernushevich, I.; Standing, K. G.; Whitman, C. P.; Kent, S. B. H. Probing the Oligomeric Structure of an Enzyme by Electrospray Ionization Time-of-Flight Mass Spectrometry. Proc. Natl. Acad. Sci. U.S.A. 1996, 93, 6851-6856.

7. Felitsyn, N.; Kitova, E. N.; Klassen, J. S. Thermal Dissociation of the Protein Homodimer Ecotin in the Gas Phase. J. Am. Soc. Mass Spectrom. 2002, 12, 1432-1442.

8. Felitsyn, N.; Kitova, E. N.; Klassen, J. S. Thermal Decomposition of a Gaseous Multiprotein Complex Studied by Blackbody Infrared Radiative Dissociation. Investigating the Origin of the Asymmetric Dissociation Behavior. Anal. Chem. 2001, 73, 4647-4661.

9. Kaleta, D. T.; Jarrold, M. F. Noncovalent Interactions Between Unsolvated Peptides. J. Phys. Chem. A 2002, 106, 9655-9664.

10. Jurchen, J. C.; Williams, E. R. Origin of Asymmetric Charge Partitioning in the Dissociation of Gas-Phase Protein Homodimers. J. Am. Chem. Soc. 2003, 125, 2817-2826.

11. Cooks, R. G.; Zhang, D. X.; Koch, K. J.; Gozzo, F. C.; Eberlin, M. N. Chiroselective Self-Directed Octamerization of Serine: Implications for Homochirogenesis. Anal. Chem. 2001, 73, 3646-3655.

12. Takats, Z.; Nanita, S. C.; Cooks, R. G. Serine Octamer Reactions: Indicators of Prebiotic Relevance. Angew. Chem. Int. Ed. 2003, 42, 3521-3523.

13. Takats, Z.; Nanita, S. C.; Cooks, R. G.; Schlosser, G.; Vekey, K. Amino Acid Clusters Formed by Sonic Spray Ionization. Anal. Chem. 2003, 75, 1514-1523.

14. Counterman, A. E.; Clemmer, D. E. Magic Number Clusters of Serine in the Gas Phase. J. Phys. Chem. B 2001, 105, 8092-8096.

15. Schalley, C. A.; Weis, P. Unusually Stable Magic Number Clusters of Serine with a Surprising Preference for Homochirality. Int. J. Mass Spectrom. 2002, 221, 9-19.

16. Scheller, M. K.; Compton, R. N.; Cederbaum, L. S. Gas-Phase Multiply-Charged Anions. Science 1995, 270, 1160-1166.

17. Dreuw, A.; Cederbaum, L. S. Multiply-Charged Anions in the Gas Phase. Chem. Rev. 2002, 102, 181-200.

18. Wang, X. B.; Wang, L. S. Observation of Negative ElectronBinding Energy in a Molecule. Nature 1999, 400, 245-248.

19. Herlert, A.; Kruckeberg, S.; Schweikhard, L.; Vogel, M.; Walther, C. First Observation of Doubly Charged Negative Gold Cluster Ions. Physica Scripta 1999, T80, T200-T202.

20. Yannouleas, C.; Landman, U.; Herlert, A.; Schweikhard, L. Multiply-Charged Metal Cluster Anions. Phys. Rev. Lett. 2001, 86, 2996-2999.

21. Yannouleas, C.; Landman, U.; Herlert, A.; Schweikhard, L. Trianionic Gold Clusters. Eur. Phys. J. D. 2001, 16, 81-85.

22. Herlert, A.; Schweikhard, L.; Vogel, M. Observation of Multiply-Charged Silver-Cluster Anions. Eur. Phys. J. D. 2001, 16, 65-68.

23. Stoermer, C.; Friedrich, J.; Kappes, M. M. Observation of Multiply-Charged Cluster Anions upon Pulsed UV Laser Ablation of Metal Surfaces Under High Vacuum. Int. J. Mass Spectrom. 2001, 206, 63-78.

24. Schauer, S. N.; Williams, P.; Compton, R. N. Production of Small Doubly Charged Negative Carbon Cluster Ions by Sputtering. Phys. Rev. Lett. 1990, 65, 625-628.

25. Gnaser, H.; Dreuw, A.; Cederbaum, L. S. Discovery of a New Class of Stable Gas-phase Dianions: Mixed Oxygen-Carbon Cluster $\mathrm{OC}_{\mathrm{n}}^{2-}(\mathrm{n}=5-19)$. J. Chem. Phys. 2002, 117, 7002-7009.

26. Gnaser, H. Formation of Gas-Phase Oxygen-Carbon Cluster $\mathrm{OC}_{\mathrm{n}}^{2-}$ Dianions by Sputtering. Phys. Rev. A 2002, 66, 013203.

27. Gozzo, F. C.; Santos, L. S.; Augusti, R.; Consorti, C. S.; Dupont, J.; Eberlin M. N. Gaseous Supramolecules of Imidazolium 
Ionic Liquids: Magic Numbers and Intrinsic Strengths of Hydrogen Bonds. Chem. Eur. J. 2004, 10, 6187-6193.

28. Hettich, R. L.; Compton, R. N.; Ritchie, R. H. Doubly-Charged Negative-Ions of Carbon-60. Phys. Rev. Lett. 1991, 67, 12421245.

29. Liu, B.; Hvelplund, P.; Brøndsted Nielsen, S.; Tomita, S. Formation of $\mathrm{C}_{60}^{2-}$ Dianions in Collisions Between $\mathrm{C}_{60}^{-}$and $\mathrm{Na}$ Atoms. Phys. Rev. Lett. 2004, 92, 168301.

30. Boltalina, O. V.; Streletskii, A. V.; Loffe, I. N.; Hvelplund, P.; Liu, B.; Brøndsted Nielsen, S.; Tomita, S. Formation of LongLived Fluorofullerene Trianions in Collisions with Na. J. Chem. Phys. 2005, 122, 021102.

31. Khairallah, G.; Peel, J. B. Identification of Dianions of $\mathrm{C}_{84}$ and $\mathrm{C}_{90}$ by Electrospray Mass Spectrometry. Chem. Phys. Lett. 1998, 296, 545-548.

32. Andersen, J. U.; Cederquist, H.; Forster, J. S.; Huber, B. A.; Hvelplund, P.; Jensen, J.; Liu, B.; Manil, B.; Maunoury, L.; Brøndsted Nielsen, S., Pedersen, U. V.; Rangama, J. ; Schmidt, H. T.; Tomita, S.; Zettergren, H. Photodissociation of Protonated Amino Acids and Peptides in an Ion Storage Ring. Determination of Arrhenius Parameters in the High Temperature Limit. Phys. Chem., Chem. Phys. 2004, 6, 2676-2681.

33. Gerlich, D. Experimental Investigations of Ion-Molecule Reactions Relevant to Interstellar Chemistry. J. Chem. Soc., Faraday Trans. 1993, 89, 2199-2208.

34. Gerlich, D. Ion-Neutral Collisions in A 22-Pole Trap at Very Low Energies. Phys. Scripta 1995, T59, T256-T263.

35. Møller, S. P. ELISA, an Electrostatic Storage Ring for Atomic Physics. Nucl. Instrum. Methods Phys. Res. A 1997, 394, 281-286.

36. Møller S. P.; Pedersen, U. V. Small Electrostatic Storage Rings; also for Highly Charged Ions? Phys. Scripta 2001, T92, T105T109.
37. Liu, B.; Tomita, S.; Rangama, J.; Hvelplund, P.; Brøndsted Nielsen, S. Electron Attachment to "Naked" and Microsolvated Nucleotide Anions: Detection of Long-Lived Dianions. Chem. Phys. Chem. 2003, 4, 1341-1344.

39. Echt, O.; Kreisle, D.; Recknagel, E.; Saenz, J. J.; Casero, R.; Soler, J. M. Dissociation Channels of Multiply-Charged van der Waals Clusters. Phys. Rev. A 1988, 38, 3236-3248.

40. Manil, B.; Maunoury, L.; Huber, B. A.; Jensen, J.; Schmidt, H. T.; Zettergren H.; Cederquist, H.; Tomita, S.; Hvelplund, P. Highly Charged Clusters of Fullerenes: Charge Mobility and Appearance Sizes. Phys. Rev. Lett. 2003, 91, 215504.

41. Concina, B., Hvelplund, P., Liu, B., Nielsen, A. B., Brøndsted Nielsen, S., Rangama, J., Tomita, S. Formation and Stability of Charged Amino Acid Clusters and the Role of Chirality. J. Am. Soc. Mass Spectrom, unpublished.

42. Hansen, K.; Andersen, J. U.; Hvelplund, P.; Møller, S. P.; Pedersen, U. V.; Petrunin, V. V. Observations of a 1/t Decay Law for Hot Clusters and Molecules in a Storage Ring. Phys. Rev. Lett. 2001, 17, 123401.

43. Andersen, J. U.; Brink, C.; Hvelplund, P.; Larsson, M. O.; Nielsen, B. B.; Shen, H. Radiative Cooling of $\mathrm{C}_{60}$. Phys. Rev. Lett. 1996, 77, 3991-3994.

44. Andersen, J. U.; Cederquist, H.; Forster, J. S.; Huber, B. A.; Hvelplund, P.; Jensen, J.; Liu, B.; Manil, B.; Maunoury, L.; Brøndsted Nielsen, S.; Pedersen, U. V.; Schmidt, H. T.; Tomita, S.; Zettergren, H. Power-Law Decay of Collisionally Excited Amino Acids and Quenching by Radiative Cooling. Eur. J. Phys. D 2003, 25, 139-148.

45. Jurchen, J. C.; Garcia, D. E.; Williams, E. R. Gas-Phase Dissociation Pathways of Multiply-Charged Peptide Clusters. J. Am. Soc. Mass Spectrom. 2003, 14, 1373-1386. 